An International Journal of Optimization and Control: Theories \& Applications ISSN:2146-0957 eISSN:2146-5703

Vol.9, No.3, pp.31-38 (2019)

http://doi.org/10.11121/ijocta.01.2019.00671

\title{
A new auxiliary function approach for inequality constrained global optimization problems
}

\author{
Nurullah Yilmaz (D) and Ahmet Sahiner [it \\ Department of Mathematics, Suleyman Demirel University, Isparta, Turkey \\ nurullahyilmaz@sdu.edu.tr, ahmetsahiner@sdu.edu.tr
}

\begin{tabular}{|c|c|}
\hline ARTICLE INFO & ABSTRACT \\
\hline $\begin{array}{l}\text { Article History: } \\
\text { Received 14 August } 2018 \\
\text { Accepted } 26 \text { January } 2019 \\
\text { Available } 15 \text { April } 2019\end{array}$ & $\begin{array}{l}\text { In this study, we deal with the nonlinear constrained global optimization prob- } \\
\text { lems. First, we introduce a new smooth exact penalty function for solving } \\
\text { constrained optimization problems. We combine the exact penalty function } \\
\text { with the auxiliary function in regard to constrained global optimization. We }\end{array}$ \\
\hline $\begin{array}{l}\text { Keywords: } \\
\text { Constrained optimization } \\
\text { Global optimization }\end{array}$ & $\begin{array}{l}\text { present a new auxiliary function approach and the adapted algorithm in order } \\
\text { to solve non-linear inequality constrained global optimization problems. Fi- } \\
\text { nally, we illustrate the efficiency of the algorithm on some numerical examples. }\end{array}$ \\
\hline
\end{tabular}

Smoothing approach

Penalty function

AMS Classification 2010:

90C26; 90C30; 65D10; 65K10

\section{Introduction}

We consider the following continuous constrained optimization problem

$$
\begin{aligned}
& \quad \min _{x \in \mathbb{R}^{n}} f(x) \\
& \text { s.t. } g_{j}(x) \leq 0, \quad j=1,2, \ldots, m,
\end{aligned}
$$

where $f: \mathbb{R}^{n} \rightarrow \mathbb{R}$ and $g_{j}(x): \mathbb{R}^{n} \rightarrow \mathbb{R}$, $j \in J=\{1,2, \ldots, m\}$ are continuously differentiable functions. The problem $(P)$ is considered in many problems of engineering and natural sciences [1-4] and it is studied in many papers [6,7].

There exists a very rich theory for the solution of the problem $(P)$ [5. One of the traditional but effective method to solve the problem $(P)$ is the penalty function method [8]. The penalty function method has been proposed in order to transform a constrained optimization problem to an unconstrained optimization problem. The method offers constructing a barrier on the boundary of the set of feasible solutions which is defined as $D_{0}:=\left\{x \in \mathbb{R}^{n}: g_{j}(x) \leq\right.$ $0, j=1,2, \ldots, m\}$ and it is assumed that $D_{0}$ is not empty. In order to construct a barrier the $" b(t)=-\log (-t) ", " b(t)=\max (t, 0)$ " functions are used. The penalized objective function is defined as

$$
F(x, \rho)=f(x)+\rho \sum_{j=1}^{m} b\left(g_{j}(x)\right),
$$

and problem $(\mathrm{P})$ re-stated as

$$
\left(P_{\rho}\right) \quad \min _{x \in \mathbb{R}^{n}} F(x, \rho),
$$

where $\rho>0$ is a penalty parameter. If $b(t)=$ $\max (t, 0)$ is in the formula (1), the penalty function is called as exact penalty function according to Zangwill [9]. It can be observed that the exact penalty function may be non-smooth. When the penalty function approach is non-smooth, one of the conventional approaches is constructing a smoothing approach. The smoothing approach is based on modifying the objective function or approximating the objective function by smooth functions [10]. In order to improve the smoothing approaches, different types of valuable techniques and algorithms are developed [11 14]. In recent years, the smoothing approaches have been used for many non-smooth problems such as min$\max [15,16]$, exact penalty [17 20] and etc. [21]. 
If the problem $(P)$ or $\left(P_{\rho}\right)$ has just one minimizer, then many local optimization methods can be used to solve with penalty method, but if it has multiple local minimizers, most of the well-known methods are not available to solve [22]. The studies on global optimization have become extensively increase among the other research areas of optimization [23, 24]. There are many valuable studies on global optimization depending on deterministic, stochastic and heuristic approaches [25, 26]. Most of the global optimization techniques are proposed to solve unconstrained problems, but by combining the penalty function method with a global optimization algorithm the global solution of the problem $(P)$ can be obtained. One of the important global optimization approaches is the auxiliary function approach which includes the Tunneling Method (Algorithm) [27, Filled Function Method [28,29], Global Descent Method [30] and Cut-Peak Function Method [31]. These methods are established on finding the lower minimizer than the current one by making a suitable modification on the objective function. The modified function is generally called as auxiliary function (Filled Function, Tunneling Function and etc.) [33.

In the next section, we give some preliminary definitions. In section 3, we introduce a new penalty function in order to transform the problem $(\mathrm{P})$ into an unconstrained problem. In Section 4, we present a minimization algorithm and convergence results. In Section 5, we apply the algorithms on the important test problems. In the last section, we give some concluding remarks.

\section{Preliminaries}

We assume that the set $D_{0}$ is closed and bounded and the function $f$ has a finite number of local minimizers in $D_{0}$. Throughout the paper, we use $x_{k}^{*}$ to denote the $k$-th local minimizer of $f$ whereas by $x^{*}$ we mean the global minimizer. $\|x\|=\sqrt{\sum_{k=1}^{n} x_{k}^{2}}$ denotes the Euclidean norm in $\mathbb{R}^{n}$.

Definition 1. [13] Let $f: \mathbb{R}^{n} \rightarrow \mathbb{R}$ be a continuous function. The function $\tilde{f}: \mathbb{R}^{n} \times \mathbb{R}_{+_{\sim}} \rightarrow \mathbb{R}$ is called a smoothing function of $f(x)$, if $\tilde{f}(\cdot, \beta)$ is continuously differentiable in $\mathbb{R}^{n}$ for any fixed $\beta$, and for any $x \in \mathbb{R}^{n}$,

$$
\lim _{z \rightarrow x, \beta \rightarrow 0} \tilde{f}(z, \beta)=f(x) .
$$

Definition 2. [19] Let $\varepsilon>0$, a point $x_{\varepsilon}$ is called $\varepsilon$-feasible solution for the problem $(P)$, if

$$
g_{j}(x) \leq \varepsilon, \quad j=1,2, \ldots, m .
$$

\section{A New Penalty Function}

In this section, we present a new penalty approach for the problem $(\mathrm{P})$. Let us define the sets $D_{j}=\left\{x \in \mathbb{R}^{n}: g_{j}(x) \leq 0\right\}$ for $j=1,2, \ldots, m$. It can be observed that $\cap_{j=1}^{m} D_{j}=D_{0}$. The main idea in exact penalty function approach is to construct a barrier at the boundary of $D_{0}$ such that any local (global) solver can not find a point outside the set $D_{0}$. Based on this idea, we define a new penalty function as

$$
F(x, \rho)=f(x)+\rho\left(\sum_{j=1}^{m} \chi_{D_{j}^{c}}(x)\right)\left\|x-x_{0}\right\|^{2},
$$

where $\rho>0, x_{0} \in D_{0}$ and

$$
\chi_{D_{j}^{c}}(x)= \begin{cases}0, & x \in D_{j}, \\ 1, & x \notin D_{j},\end{cases}
$$

for $j=1,2, \ldots, m$. Since the function $\chi_{D_{j}^{c}}(x)$ is non-smooth, we apply the smoothing approach to this function in order to make it smooth. We design the following function

$$
\tilde{\chi}_{D_{j}^{c}}(x, \varepsilon)= \begin{cases}0, & t \leq 0 \\ R_{1}(t), & 0 \leq t \leq \varepsilon \\ 1, & t \geq \varepsilon\end{cases}
$$

where $\varepsilon>0$ and

$$
R_{1}(t)=\frac{-2}{\varepsilon^{3}} t^{3}+\frac{3}{\varepsilon^{2}} t^{2},
$$

for $t=g_{j}(x), j=1,2, \ldots, m$. By using $R_{1}$ in formula (2), the obtained smoothing function is continuously differentiable. If the following function

$$
R_{2}(t)=\frac{6}{\varepsilon^{5}} t^{5}-\frac{15}{\varepsilon^{4}} t^{4}+\frac{10}{\varepsilon^{3}} t^{3},
$$

is used in formula (2) instead of $R_{1}$, the obtained smoothing function is second order continuously differentiable. The function $R_{i},(i=1,2, \ldots, k)$ is called the smooth transition function. Now, we obtain surrogate problem $\left(\tilde{P}_{\rho}\right)$ as follows:

$$
\left(\tilde{P}_{\rho}\right) \quad \min _{x \in \mathbb{R}^{n}} F(x, \rho, \varepsilon),
$$

where

$F(x, \rho, \varepsilon)=f(x)+\rho\left(\sum_{j=1}^{m} \tilde{\chi}_{D_{j}^{c}}(x, \varepsilon)\right)\left\|x-x_{0}\right\|^{2}$.

Theorem 1. Let $x^{*}$ be a solution for $\left(\tilde{P}_{\rho}\right)$ for sufficiently large $\rho>0$ then $x^{*} \in D_{0}$.

Proof. Suppose that $x^{*} \notin D_{0}$. Then, there exists $j$ such that $t=g_{j}\left(x^{*}\right)>0$. We have two cases:

Case 1. Let $t \geq \varepsilon$ then, we have

$$
F\left(x^{*}, \rho, \varepsilon\right)=f\left(x^{*}\right)+\rho\left\|x^{*}-x_{0}\right\|^{2},
$$

and

$$
\nabla F\left(x^{*}, \rho, \varepsilon\right)=\nabla f\left(x^{*}\right)+2 \rho\left(x^{*}-x_{0}\right)=0 .
$$


Therefore, we obtain

$$
\rho=-\nabla f\left(x^{*}\right)\left(2\left(x^{*}-x_{0}\right)\right)^{-1} .
$$

Since $f(x)$ continuous differentiable $\|f(x)\|<\infty$ and $x^{*} \neq x_{0}$, it can be concluded that $\rho$ is finite. If anyone chooses $\rho_{1}>\rho$, the $\nabla F\left(x^{*}, \rho_{1}, \varepsilon\right) \neq 0$.

Case 2. Let $0<t \leq \varepsilon$ then, we have

$$
\begin{aligned}
F\left(x^{*}, \rho, \varepsilon\right) & =f\left(x^{*}\right)+\rho\left(\frac{-2}{\varepsilon^{3}} t^{3}+\frac{3}{\varepsilon^{2}} t^{2}\right) \\
& \times\left\|x^{*}-x_{0}\right\|^{2},
\end{aligned}
$$

and

$$
\nabla F\left(x^{*}, \rho, \varepsilon\right)=\nabla f\left(x^{*}\right)+\rho A\left(x^{*}, \varepsilon\right),
$$

where

$$
\begin{aligned}
A\left(x^{*}, \varepsilon\right)= & \left(\left(\frac{-2}{\varepsilon^{3}} t^{3}+\frac{3}{\varepsilon^{2}} t^{2}\right)\left\|x^{*}-x_{0}\right\|^{2}\right. \\
& \left.+\left(\frac{-2}{\varepsilon^{3}} t^{3}+\frac{3}{\varepsilon^{2}} t^{2}\right)\left(2\left(x^{*}-x_{0}\right)\right)\right) .
\end{aligned}
$$

Thus, we obtain

$$
\rho=-\nabla f\left(x^{*}\right) A\left(x^{*}, \varepsilon\right)^{-1} .
$$

It can be seen that $\rho$ is finite. If anyone chooses $\rho_{2}>\rho$, the $\nabla F\left(x^{*}, \rho_{2}, \varepsilon\right) \neq 0$.

As a consequence, if anyone chose the parameter $\rho$ in (1) as $\rho>\max \left\{\rho_{1}, \rho_{2}\right\}$, the point $x^{*}$ cannot be outside of $D_{0}$.

Corollary 1. Let $x^{*}$ be a solution for $\left(\tilde{P}_{\rho}\right)$ for sufficiently large $\rho$ then $x^{*}$ is a solution for $(P)$.

Proof. From Theorem 1, we have $x^{*} \in D_{0}$. Then, we obtain

$$
\begin{aligned}
f\left(x^{*}\right) & =F\left(x^{*}, \rho, \varepsilon\right) \\
& =F\left(x^{*}, \rho, 0\right) \\
& \leq F(x, \rho, 0) \\
& =f(x) .
\end{aligned}
$$

This completes the proof.

\section{Algorithms for Minimization Procedure}

In this section, we propose our new algorithm to find the global optimal point by considering the problem $\left(\tilde{P}_{\rho}\right)$.

\section{Algorithm}

Step 1 Determine $x^{0}, \rho_{0}=10, \varepsilon_{0}>0, N>1$, $0<\eta<1$ and let $j=1$ and go to Step 2 .

Step 2 Use $x^{j-1}$ as an initial point and apply one of the global optimization algorithms to solve the problem $\left(\tilde{P}_{\rho}\right)$. Let $x_{j}$ is the solution.

Step 3 If $x_{j} \in \operatorname{int} D_{0}$ then stop the algorithm and $x^{j}$ is the optimal solution else go to Step 4 .
Step 4 If $x^{j}$ is $\varepsilon$-feasible for $(P)$, then stop and $x^{j}$ is the optimal solution. Otherwise, take $\rho_{j}=N \rho_{j-1}, \varepsilon_{j}=\eta \varepsilon_{j-1}$ and $j=$ $j+1$, then go to Step 2 .

In Step 2 of algorithm $x_{j}$ is the global optimal solution of the problem $\left(\tilde{P}_{\rho}\right)$ depending on the parameter $\varepsilon$. In order to obtain the global solution, any of the global optimization methods can be used. We use the auxiliary function based global optimization method studied in [21,33]. The Auxiliary Function Method (AFM) is very effective in terms of numerical results which is illustrated in [21]. Our auxiliary function is defined as follows:

$$
\begin{aligned}
\tilde{\phi}\left(x, x_{k}^{*}, \beta, \alpha\right)= & f_{k}^{*}+\left(f(x)-f_{k}^{*}\right) \tilde{\chi}_{A_{k}}(t, \beta) \\
& +\alpha H\left(\left\|x-x_{k}^{*}\right\|^{2}\right),
\end{aligned}
$$

where $\alpha$ and $\beta$ are real parameters. The function $\tilde{\chi}_{A_{k}}(t, \beta)$ is defined by

$$
\tilde{\chi}_{A_{k}}(t, \beta)= \begin{cases}0, & t>\beta, \\ q(t, \beta), & -\beta \leq t \leq \beta, \\ 1, & t<-\beta,\end{cases}
$$

where

$$
q(t, \beta)=\frac{1}{4 \beta^{3}} t^{3}-\frac{3}{4 \beta} t+\frac{1}{2},
$$

and the function $H$ is defined on $\mathbb{R}_{+}$and it satisfies the following properties:

$$
\begin{aligned}
& \text { i. } H(u)>0, \\
& \text { ii. } H^{\prime}(u)<0, \\
& \text { iii. } \lim _{u \rightarrow \infty} H(u)=0 .
\end{aligned}
$$

At Step 3 and 4, the feasibility of the solution is checked and the stopping conditions are declared. In order to guarantee that the algorithm is worked straightly, we prove the following theorems.

Theorem 2. Assume that the sequence $\left\{x^{j}\right\}$ is produced by the Algorithm has a limit point $x^{*}$, then $x^{*} \in D_{0}$.

Proof. Assume $x^{*}$ is a limit point of $\left\{x^{j}\right\}$. Then there exists set $J \subset \mathbb{N}$, such that $x^{j} \rightarrow \bar{x}$ for $j \in J$. Let us consider the contrary that $x^{*} \notin D_{0}$, i.e. for sufficiently large $j \in J$, there exist $\delta_{0}>0$ and $i_{0} \in\{1,2, \ldots, m\}$ such that:

Case 1. $g_{i_{0}}\left(x^{j}\right) \geq \delta_{0} \geq \varepsilon>0$. Since $x^{j}$ is the global minimum according $j$-th values of the parameters $\rho_{j}, \varepsilon_{j}$, for any $x \in D_{0}$ we have

$$
F\left(x^{j}, \rho_{j}, \varepsilon_{j}\right)=f\left(x^{j}\right)+\rho_{j}\left\|x^{j}-x_{0}\right\| .
$$

If $j \rightarrow \infty$ then, $\rho_{j} \rightarrow \infty$ and $\rho_{j}\left\|x^{j}-x_{0}\right\| \rightarrow \infty$ (since $x^{j} \notin D_{0}$ and $\left\|x^{j}-x_{0}\right\|>0$ ). Thus, $f(x)$ takes infinite values on $D_{0}$ and it contradicts with the boundedness of $f$ on $D_{0}$.

Case 2. $t=g_{i_{0}}\left(x^{j}\right) \geq \varepsilon \geq \delta_{0}>0$. Since $x^{j}$ is the global minimum according to $j$-th values of the 
parameters $\rho_{j}, \varepsilon_{j}$, for any $x \in D_{0}$ we have

$$
\begin{aligned}
F\left(x^{j}, \rho_{j}, \varepsilon_{j}\right) & =f\left(x^{j}\right)+\rho_{j}\left(\frac{-2}{\varepsilon^{3}} t^{3}+\frac{3}{\varepsilon^{2}} t^{2}\right)\left\|x^{j}-x_{0}\right\| \\
& \geq f\left(x^{j}\right)+\rho_{j}\left\|x^{j}-x_{0}\right\| .
\end{aligned}
$$

If $j \rightarrow \infty$ then, $\rho_{j} \rightarrow \infty$ and $\rho_{j}\left\|x^{j}-x_{0}\right\| \rightarrow \infty$ (since $x^{j} \notin D_{0}$ and $\left\|x^{j}-x_{0}\right\|>0$ ). Thus, $f(x)$ takes infinite values on $D_{0}$ and it contradicts with the boundedness of $f$ on $D_{0}$. From the Cases 1 and 2 , we obtain the result.

Theorem 3. Assume that for $\varepsilon \in\left(0, \varepsilon_{0}\right]$ the set

$$
\underset{x \in \mathbb{R}^{n}}{\operatorname{argmin}} F(x, \rho, \varepsilon) \neq \emptyset .
$$

Let $x^{j}$ is generated by Algorithm when $\eta N<1$. If $\left\{x^{j}\right\}$ has a limit point, then the limit point of $x^{j}$ is the solution for $(P)$.

Proof. Let $x^{*}$ be a limit point of $\left\{x^{j}\right\}$. From Theorem 2, we have $x^{*} \in D_{0}$. Then, we obtain

$$
\begin{aligned}
f\left(x^{*}\right) & =F\left(x^{*}, \rho, \varepsilon\right) \\
& =F\left(x^{*}, \rho, 0\right) \\
& \leq F(x, \rho, 0) \\
& =f(x) .
\end{aligned}
$$

This completes the proof.

\section{Numerical Examples}

In this section, we apply our algorithm to test problems. The proposed algorithm is programmed in Matlab. Numerical results show the efficiency of this method. The detailed results are presented in the tables for all problems. For these tables, we use some symbols in order to abbreviate the expressions. The meanings of these symbols are as follows:

$j$ :The number of iterations,

$x^{j}$ :the local minimum point of the $j$ th iteration,

$\varepsilon_{j}$ :smoothing parameter of the $j$ th iteration,

$g\left(x^{j}\right)$ :the value of the point $x^{j}$ under the constraint functions,

$F\left(x^{j}, \rho_{j}, \varepsilon_{j}\right)$ :the value of the point $x^{j}$ under $F$, $f\left(x^{j}\right)$ :the value of the point $x^{j}$ under $f$.

Problem 1. Let us consider the Example in 34] $\min f(x)=x_{1}^{2}+x_{2}^{2}-\cos \left(17 x_{1}\right)-\cos \left(17 x_{2}\right)+3$,

s.t. $\quad g_{1}(x)=\left(x_{1}-2\right)^{2}+x_{2}^{2} \leq 1.6^{2}$, $g_{2}(x)=x_{1}^{2}+\left(x_{2}-3\right)^{2} \leq 2.7^{2}$, $0 \leq x_{1} \leq 2, \quad 0 \leq x_{2} \leq 2$.
We choose $x^{0}=(1,1)$ as a starting point $\rho_{0}=$ 10, $\varepsilon_{0}=0.01, \eta_{0}=0.1$ and $N=3$. The results are shown in the Table 1 . Considering $\left(\tilde{P}_{\rho}\right)$ the global minimum is obtained at a point $x^{*}=(0.7254,0.3993)$ with the corresponding value 1.8376. In the paper [34], the obtained global minimum point is $x^{*}=(0.72540669,0.3992805)$ with the corresponding value 1.837623 . Our algorithm finds the correct point as in 34].

Problem 2. Let us consider the Example in [35]

$$
\begin{aligned}
& \min f(x)=-x_{1}-x_{2}, \\
& \text { s.t. } x_{2}-2 x_{1}^{4}+8 x_{1}^{3}-6 x_{1}^{2} \leq 2, \\
& \quad x_{2}-4 x_{1}^{4}+32 x_{1}^{3}-88 x_{1}^{2}+96 x_{1} \leq 36, \\
& 0 \leq x_{1} \leq 3, \quad 0 \leq x_{2} \leq 4 .
\end{aligned}
$$

We choose $x^{0}=(0,0)$ as a starting point $\rho_{0}=10$, $\varepsilon_{0}=0.01, \eta_{0}=0.1$ and $N=3$. The results are shown in the Table 2. The global minimum is obtained at a point $x^{*}=(2.3295,3.1783)$ with the corresponding value -5.5079 . In the papers [35, 36], the obtained global minimum point is $x^{*}=(2.3289,3.1883)$ with the corresponding value -5.5091. Our algorithm find the correct point as in [35, 36].

Problem 3. Let us consider the example in [34], $\min f(x)=1000-x_{1}^{2}-2 x_{2}^{2}-x_{3}^{2}-x_{1} x_{2}-x_{1} x_{3}$,

$$
\begin{aligned}
\text { s.t. } g_{1}(x) & =\sum_{i=1}^{3} x_{i}^{2}=25 \\
g_{2}(x) & =\left(x_{1}-5\right)^{2}+\sum_{i=2}^{3} x_{i}^{2}=25, \\
g_{3}(x) & =\sum_{i=1}^{3}\left(x_{i}-5\right)^{2} \leq 25 .
\end{aligned}
$$

We choose $x^{0}=(2,2,2)$ as a starting point $\rho_{0}=$ $10, \varepsilon_{0}=0.01, \eta_{0}=0.1$ and $N=3$. The results are shown in the Table 3 . The global minimum is obtained at a points $x^{*}=(2.5000,4.2196,0.9721)$ with the corresponding value 944.2157 . In the papers [34], the obtained global minimum point is $x^{*}=(2.500000,4.221305,0.964666)$ with the corresponding value 944.2157. Our algorithm finds the correct solution with the lower iteration numbers in comparison with the algorithm in [34].

Problem 4. Consider the example in [36],

$$
\begin{aligned}
\min f(x)= & -x_{1}^{2}+x_{2}^{2}+x_{3}^{2}-x_{1}, \\
\text { s.t. } \quad & g_{1}(x)=x_{1}^{2}+x_{2}^{2}+x_{3}^{2} \leq 4, \\
& g_{2}(x)=\min \left\{x_{2}-x_{3}, x_{3}\right\} \leq 0 .
\end{aligned}
$$

We choose $x^{0}=(-1.6,-1,0.2)$ as a starting point $\rho_{0}=10, \varepsilon_{0}=0.01, \eta_{0}=0.1$ and 
Table 1. Table of minimization process of the Problem 1.

\begin{tabular}{cccccccc}
\hline$j$ & $x^{j}$ & $\rho_{j}$ & $\varepsilon_{j}$ & $g_{1}\left(x^{j}\right)$ & $g_{2}\left(x^{j}\right)$ & $F\left(x^{j}, \rho_{j}, \varepsilon_{j}\right)$ & $f\left(x^{j}\right)$ \\
\hline \hline 1 & $(0.7249,0.4007)$ & 10 & 0.01 & -0.7737 & -0.0083 & 1.8774 & 1.8522 \\
2 & $(0.7252,0.3996)$ & 30 & 0.001 & -0.7753 & -0.0018 & 1.8446 & 1.8408 \\
3 & $(0.7253,0.3993)$ & 90 & 0.0001 & -0.7758 & -0.0003 & 1.8388 & 1.8382 \\
4 & $(0.7253,0.3992)$ & 270 & $1 e-05$ & -0.7758 & $-6.2645 e-05$ & 1.8378 & 1.8377 \\
5 & $(0.7253,0.3992)$ & 810 & $1 e-06$ & -0.7758 & $-1.0667 e-05$ & 1.8376 & 1.8376 \\
\hline
\end{tabular}

Table 2. Table of minimization process of the Problem 2.

\begin{tabular}{cccccccc}
\hline$j$ & $x^{j}$ & $\rho_{j}$ & $\varepsilon_{j}$ & $g_{1}\left(x^{j}\right)$ & $g_{2}\left(x^{j}\right)$ & $F\left(x^{j}, \rho_{j}, \varepsilon_{j}\right)$ & $f\left(x^{j}\right)$ \\
\hline \hline 1 & $(2.3307,3.1477)$ & 10 & 0.01 & 3.9592 & 508.57 & -5.4454 & -5.4784 \\
2 & $(2.3297,3.173)$ & 30 & 0.001 & 3.9928 & 507.95 & -5.4973 & -5.5027 \\
3 & $(2.3296,3.1775)$ & 90 & 0.0001 & 3.9988 & 507.85 & -5.5062 & -5.5071 \\
4 & $(2.3295,3.1783)$ & 270 & $1 e-05$ & 3.9998 & 507.83 & -5.5079 & -5.5079 \\
\hline
\end{tabular}

Table 3. Table of minimization process of the Problem 3.

\begin{tabular}{ccccccccc}
\hline$j$ & $x^{j}$ & $\rho_{j}$ & $\varepsilon_{j}$ & $g_{1}\left(x^{j}\right)$ & $g_{2}\left(x^{j}\right)$ & $g_{3}\left(x^{j}\right)$ & $F_{p}\left(x^{j}, \rho_{j}, \varepsilon_{j}\right)$ & $f\left(x^{j}\right)$ \\
\hline \hline 1 & $(2.5002,4.2214,0.9650)$ & 10 & 0.01 & 0.0022 & 0.0001 & -1.864 & 944.4134 & 944.2108 \\
2 & $(2.5000,4.2212,0.9650)$ & 30 & 0.001 & $7.17 e-05$ & $3.26 e-06$ & -1.8625 & 944.2756 & 944.2155 \\
3 & $(2.5000,4.2212,0.9650)$ & 90 & 0.0001 & $1.73 e-05$ & $-2.62 e-05$ & -1.8625 & 944.2341 & 944.2156 \\
4 & $(2.5000,4.2212,0.9650)$ & 270 & $1 e-05$ & $3.92 e-06$ & $-4.27 e-06$ & -1.8625 & 944.2157 & 944.2157 \\
\hline
\end{tabular}

Table 4. Table of minimization process of the Problem 4.

\begin{tabular}{cccccccc}
\hline$j$ & $x^{j}$ & $\rho_{j}$ & $\varepsilon_{j}$ & $g_{1}\left(x^{j}\right)$ & $g_{2}\left(x^{j}\right)$ & $F\left(x^{j}, \rho_{j}, \varepsilon_{j}\right)$ & $f\left(x^{j}\right)$ \\
\hline \hline 1 & $(1.995,-0.0300,0.0300)$ & 10 & 0.01 & -0.0180 & -0.0601 & -5.9393 & -5.9733 \\
2 & $(1.9991,-0.0094,0.0094)$ & 30 & 0.001 & -0.0033 & -0.0188 & -5.9902 & -5.9954 \\
3 & $(1.9998,-0.0029,0.0029)$ & 90 & 0.0001 & -0.0005 & -0.0058 & -5.9984 & -5.9992 \\
4 & $(2.0000,-0.0009,0.0009)$ & 270 & $1 e-05$ & -0.0001 & -0.0018 & -5.9997 & -5.9999 \\
5 & $(2.0000,-0.0009,0.0009)$ & 810 & $1 e-06$ & $-1.6 e-05$ & -0.0018 & -6.0000 & -6.0000 \\
\hline
\end{tabular}

$N=3$. The results are shown in the Table 4. The global minimum is obtained at a point $x^{*}=(2,-0.0009,0.0009)$ with the corresponding value -6.0000 . In the papers [35, [36], the obtained global minimum point is $x^{*}=$ $(1.9889,-0.0001,-0.0111)$ with the corresponding value -5.9446. Our algorithm finds the correct point as in [35, 36].

Problem 5. The Rosen-Suzuki problem in [34]

$$
\begin{aligned}
& \min f(x)=\sum_{i=1}^{4} x_{i}^{2}-5 x_{1}-21 x_{3}+7 x_{4}, \\
& \text { s.t. } g_{1}(x)=2 x_{1}^{2}+\sum_{i=2}^{3} x_{i}^{2}+2 x_{1}+x_{2}+x_{4} \leq 5, \\
& g_{2}(x)=\sum_{i=1}^{4} x_{i}^{2}+x_{1}-x_{2}+x_{3}-x_{4} \leq 8,
\end{aligned}
$$

$$
g_{3}(x)=\sum_{i=1}^{2}\left(x_{2 i-1}^{2}+2 x_{2 i}^{2}\right)-x_{1}-x_{4} \leq 10 .
$$

First, we choose $x^{0}=(0,0,0,0), \rho_{0}=10$, $\varepsilon_{0}=0.01, \eta_{0}=0.1$ and $N=3$. The results are shown in the Tables 5. The global minimum is obtained at a point $x^{*}=$ $(0.1697,0.8358,2.0084,-0.9651)$ with the corresponding value -44.2338. In the paper [19], the obtained global minimum point is $x^{*}=$ (0.1684621, 0.8539065, 2.000167, -0.9755604) with the corresponding value -44.23040 . In [34], the obtained global minimum point is $x^{*}=$ $(0.170189,0.835628,2.008242,-0.95245)$ with the corresponding value -44.2338 . It can be seen that our algorithm present numerically better result than the algorithm in 34]. 
Table 5. Table of minimization process of the Problem 5.

\begin{tabular}{ccccccccc}
\hline$j$ & $x^{j}$ & $\rho_{j}$ & $\varepsilon_{j}$ & $g_{1}\left(x^{j}\right)$ & $g_{2}\left(x^{j}\right)$ & $g_{3}\left(x^{j}\right)$ & $F\left(x^{j}, \rho_{j}, \varepsilon_{j}\right)$ & $f\left(x^{j}\right)$ \\
\hline \hline 1 & $(0.1682,0.8338,2.0070,-0.9661)$ & 10 & 0.01 & -0.0161 & -0.0075 & -1.8886 & -44.1675 & -44.2068 \\
2 & $(0.1694,0.8351,2.0083,-0.9651)$ & 30 & 0.001 & -0.0030 & -0.0017 & -1.885 & -44.2217 & -44.2281 \\
3 & $(0.1697,0.8354,2.0085,-0.9649)$ & 90 & 0.0001 & -0.0005 & -0.0003 & -1.8839 & -44.2317 & -44.2328 \\
4 & $(0.1697,0.8354,2.0086,-0.9649)$ & 270 & $1 e-05$ & $-9.36 e-05$ & $-5.85 e-05$ & -1.8837 & -44.2335 & -44.2337 \\
5 & $(0.1697,0.8354,2.0086,-0.9649)$ & 810 & $1 e-06$ & $-5.60 e-05$ & $-1.20 e-05$ & -1.8837 & -44.2338 & -44.2338 \\
\hline
\end{tabular}

Problem 6. Consider the example in [35],

$$
\begin{aligned}
\min f(x) & =\frac{\pi}{n}\left[10 \sin ^{2} \pi x_{1}+h(x)+\left(x_{n}-1\right)^{2}\right], \\
\text { s.t. } & -10 \leq x_{i} \leq 10 \quad i=1,2, \ldots, n,
\end{aligned}
$$

where $h(x)=\sum_{i=1}^{n-1}\left[\left(x_{i}-1\right)^{2}\left(1+10 \sin ^{2} \pi x_{i+1}\right)\right]$. For $n=3,5,7$ we choose $x^{0}=(6,6, \ldots, 6)$ as a starting point $\rho_{0}=10, \varepsilon_{0}=0.01, \eta_{0}=0.1$ and $N=3$. The results are shown in the Table 6. The global minimum is obtained at a point $x^{*}=(1,1, \ldots, 1)$ with the corresponding value 0 . In the paper [35], the obtained global minimum point is $x^{*}=(1,1, \ldots, 1)$ with the corresponding value 0 . Our algorithm finds the correct point as in [35].

Problem 7. Let us consider the Example in [34]

$$
\begin{aligned}
\min f(x) & =10 x_{2}+2 x_{3}+x_{4}+3 x_{3}+4 x_{6}, \\
\text { s.t. } & g_{1}(x)=x_{1}+x_{2}=10, \\
& g_{2}(x)=-x_{1}+x_{3}+x_{4}+x_{5}=0, \\
& g_{3}(x)=-x_{2}-x_{3}+x_{5}+x_{6}=0, \\
& g_{4}(x)=10 x_{1}-2 x_{3}+3 x_{4}-2 x_{5} \leq 16, \\
& g_{5}(x)=x_{1}+4 x_{3}+x_{5} \leq 10, \\
& 0 \leq x_{1} \leq 12,0 \leq x_{2} \leq 18, \\
& 0 \leq x_{3} \leq 5,0 \leq x_{4} \leq 12, \\
& 0 \leq x_{5} \leq 1,0 \leq x_{6} \leq 16 .
\end{aligned}
$$

We choose $x^{0}=(0,0, \ldots, 0)$ as a starting point $\rho_{0}=10, \varepsilon_{0}=0.01, \eta_{0}=0.1$ and $N=4$ for the Algorithm. The results are shown in the Table 7. In [34], in which three algorithms are offered for a new smoothing technique, approximate solution is found with 4, 3 and 13 iterations in the Algorithms I, II and III, respectively. Note that the solution is not found in Algorithm II of [34]. Whereas, an approximate solution is found with 4 iterations in our Algorithm.

\section{Conclusion}

In this study, we propose a new exact penalty function and a new algorithm for continuous constrained optimization. By considering this new penalty function approach, we construct a new minimization algorithm. We apply the algorithm on test problems and obtain satisfactory results.
We also propose a new smoothing approach for non-smooth penalty functions and it provides good approximations to the non-smooth penalty functions. Moreover, it is easy applicable and has easy formulation.

The results convince that the Algorithm can be used for large scale optimization problems. By applying the minimization algorithm, the optimum value is found rapidly and the algorithm presents high accuracy in finding the optimum point. We use the auxiliary function method in the algorithm as a global optimizaiton method but anyone can use any other algorithms such as DIRECT [38], Kriging-based techniques [39] or heuristic algorithms [40,41].

\section{References}

[1] Ling, B.W.K., Wu, C.Z., Teo, K.L. \& Rehbock, V. (2013). Global optimal design of IIR filters via constraint transcription and filled function methods. Circuits, Systems, and Signal Processing, 32, 1313-1334.

[2] Evirgen, F. (2016). Analyze the optimal solutions of optimization problems by means of fractional gradient based system using VIM. An International Journal of Optimization and Control: Theories \& Applications (IJOCTA). 6(2), 75-83.

[3] Akteke-Ozkurt, B., Weber, G.W. and Koksal, G. (2017). Optimization of generalized desirability functions under model uncertainty. Optimization, 66(12), 2157-2169.

[4] Ozmen, A., Kropat, E. \& Weber, G.W. (2017). Robust optimization in spline regression models for multi-model regulatory networks under polyhedral uncertainty. Optimization, 66 (12), 2135-2155.

[5] Nocedal, J. and Wright, S.J. (2006). Numerical Optimization. 2nd Edition, Springer, New York.

[6] Di Pillo, G. \& Grippo, L. (1989). Exact penalty functions in constrained optimization, SIAM Journal on Control and Optimization, 27(6), 1333-1360.

[7] Zheng, F.Y. \& Zhang, L.S. (2012). New simple exact penalty function for constrained 
Table 6. Table of minimization process of the Problem 6.

\begin{tabular}{ccccccc}
\hline \hline$n$ & $j$ & $x^{j}$ & $\rho_{j}$ & $\varepsilon_{j}$ & $F\left(x^{j}, \rho_{j}, \varepsilon_{j}\right)$ & $f\left(x^{j}\right)$ \\
\hline \multirow{2}{*}{3} & 1 & $(1.0000,1.0000,1.0000)$ & 10 & 0.01 & $5.8448 e-13$ & $5.8448 e-13$ \\
& 2 & $(1.0000,1.0000,1.0000)$ & 30 & 0.001 & $5.7929 e-13$ & $5.7929 e-13$ \\
\hline \multirow{2}{*}{5} & 1 & $(1.0000,1.0000, \ldots, 1.0000)$ & 10 & 0.01 & $1.6646 e-12$ & $1.6646 e-12$ \\
& 2 & $(1.0000,1.0000, \ldots, 1.0000)$ & 30 & 0.001 & $1.4643 e-12$ & $1.4643 e-12$ \\
\hline \multirow{2}{*}{7} & 1 & $(1.0000,1.0000, \ldots, 1.0000)$ & 10 & 0.01 & $2.5965 e-14$ & $2.5965 e-14$ \\
& 2 & $(1.0000,1.0000, \ldots, 1.0000)$ & 30 & 0.001 & $1.1379 e-15$ & $1.1379 e-15$ \\
\hline
\end{tabular}

Table 7. Table of minimization process of the Problem 7.

\begin{tabular}{|c|c|c|c|c|c|c|c|c|c|c|}
\hline$j$ & $x^{j}$ & $\rho_{j}$ & $\varepsilon_{j}$ & $g_{1}\left(x^{j}\right)$ & $g_{2}\left(x^{j}\right)$ & $g_{3}\left(x^{j}\right)$ & $g_{4}\left(x^{j}\right)$ & $g_{5}\left(x^{j}\right)$ & $F\left(x^{j}, \rho_{j}, \varepsilon_{j}\right)$ & $f\left(x^{j}\right)$ \\
\hline 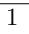 & 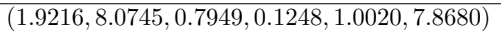 & 40 & 0.001 & -0.0039 & $-5.33 e-07$ & 0.0006 & -0.0032 & -3.8969 & 117.2 & 116.9372 \\
\hline 2 & $(1.9208,8.0792,0.7945,0.1265,0.9998,7.8739)$ & 160 & 0001 & $-8.99 e-06$ & $-2.56 e-06$ & $-2.57 e-06$ & -0.0007 & -3.9015 & 117.0525 & 117.0021 \\
\hline 3 & $(1$ & 640 & $1 e-05$ & $71 e-$ & $-2.49 e$ & $-1.76 e$ & -0 & -3 & 117 & 117.0022 \\
\hline 4 & $(1.9208,8.0792,0.7945,0.1265,0.9998,7.8739)$ & 2560 & $1 e-06$ & $-3.05 e-07$ & $2.79 e-08$ & $5.72 e-09$ & -0.0006 & -3.9015 & 117.0100 & 117.0022 \\
\hline
\end{tabular}

optimization. Applied Mathematics and Mechanics, 33(7), 951-962.

[8] Rao, S.S. (2009). Engineering Optimization: Theory and Practice. 4th Edition, John Wiley \& Sons, New Jersey.

[9] Zangwill, W.I. (1967). Nonlinear programing via penalty functions. Management Science, $13,344-358$.

[10] Bertsekas, D. (1975). Nondifferentiable optimization via approximation. Mathematical Programming Study, 3, 1-25.

[11] Chen, C., \& Mangasarian, O.L. (1996). A class of smoothing functions for nonlinear and mixed complementarity problem. Computational Optimization and Applications, 5, 97138.

[12] Xavier, A.E. (2010). The hyperbolic smoothing clustering method. Pattern Recognition, 43, 731-737.

[13] Chen, X. (2012). Smoothing methods for nonsmooth, nonconvex minimzation. Mathematical Programming Ser. B, 134 71-99.

[14] Grossmann, C. (2016). Smoothing techniques for exact penalty function methods. Contemporary Mathematics, 658 , 249-265.

[15] Zang, I. (1980). A smoothing out technique for min-max optimization. Mathematical Programming, 19, 61-77.

[16] Bagirov, A.M., Nuamiat, A. Al \& Sultanova, N. (2013). Hyperbolic smoothing functions for non-smooth minimization. Optimization, 62(6), 759-782.

[17] Pinar, M.C. \& Zenios, S. (1994). On smoothing exact penalty functions for convex constrained optimization. SIAM Journal of $\mathrm{Op}$ timization, 4, 468-511.
[18] Meng, Z., Dang, C., Jiang, M. \& Shen R. (2011). A smoothing objective penalty function algorithm for inequality constrained optimization problems. Journal Numerical Functional Analysis and Optimization, 32, 806820.

[19] Lian, S.J. (2012). Smoothing approximation to $l_{1}$ exact penalty for inequality constrained optimization. Applied Mathematics and Computation, 219, 3113-3121 (2012).

[20] Sahiner, A., Kapusuz, G. \& Yilmaz, N. (2016). A new smoothing approach to exact penalty functions for inequality constrained optimization problems. Numerical Algebra, Control and Optimization, 6(2), 161-173.

[21] Sahiner, A., Yilmaz, N. \& Kapusuz, G. (2019). A novel modeling and smoothing technique in global optimization. Journal of Industrial and Management Optimization, 15 (1), 113-139.

[22] Lin, H., Wang, Y., Gao, Y. \& Wang, X. (2018). A filled function method for global optimization with inequality constraints. Computational and Applied Mathematics, 37(2), 1524-1536.

[23] Horst, R. \& Pardalos, P.M. (eds.). (1995). Handbook of Global Optimization. Kluwer Academic Publishers, Dordrecht.

[24] Locatelli, M. \& Schoen, F. (2013). Global Optimization: Theory, Algorithms, and Applications. SIAM, Philadelphia.

[25] Sergeyev, Y.D., Strongin, R.G. \& Lera, D. (2013). Introduction to Global Optimization Exploiting Space-Filling Curves. Springer, New York.

[26] Zhigljavsky, A. \& Zilinskas, A. (2008). Stochastic Global Optimization, Springer, New York.

[27] Levy, A.V. \& Montalvo, A. (1985). The tunneling algorithm for the global minimization 
of functions. SIAM Journal on Scientific and Statistical Computing, 6 (2), 15-29.

[28] Ge, R.P. (1990). A filled function method for finding global minimizer of a function of several variables. Mathematical Programming, 46, 191-204.

[29] Zhang, L.-S., Ng, C.-K., Li, D. \& Tian, W.W. (2004). A new filled function method for global optimization. Journal of Global Optimization, 28, 17-43.

[30] Ng, C.K., Li, D. \& Zhang, L.S. (2010). Global descent method for global optimization. SIAM Journal of Optimization, 20(6), 3161-3184.

[31] Wang, Y., Fang, W. \& Wu, T. (2009). A cutpeak function method for global optimization. Journal of Computational and Applied Mathematics, 230, 135-142.

[32] Liu, J., Zhang, S., Wu, C., Liang, J., Wang, X. \& Teo, K.L. (2016). A hybrid approach to constrained global optimization. Applied Soft Computing, 47, 281-294.

[33] Sahiner, A., Yilmaz, N. \& Kapusuz, G. (2017). A descent global optimization method based on smoothing techniques via Bezier curves. Carpathian Journal of Mathematics, 33(3), 373-380.

[34] Xu, X., Meng, Z., Sun, J. \& Shen, R. (2011). A penalty function method based on smoothing lower order penalty function. Journal of Computational and Applied Mathematics, 235, 4047-4058.

[35] Zhang, Y., Xu, Y. \& Zhang, L. (2009). A filled function method applied to nonsmooth constrained global optimization. Journal of
Computational and Applied Mathematics, 232, 415-426.

[36] Wu, Z.Y., Bai, F.S., Lee, H.W.J. \& Yang, Y.J. (2007). A filled function method for constrained global optimization. Journal of Global Optimization, 39, 495-507.

[37] Gao, Y., Yang, Y. \& You, M. (2015). A new filled function method for global optimization. Applied Mathematics and Computation, 268, 685-695.

[38] Jones, D.R., Perttunen, C.D. \& Stuckman, B.E. (1993). Lipschitzian optimization without the Lipschitz constant. Journal of Optimization Theory and Applications, 79, 157181.

[39] Jones, D.R., Schonlau, M. \& Welch, W.J. (1998). Efficient global optimization of expensive black-box functions. Journal of Global Optimization, 13, 455-492.

[40] Kirkpatrick, S., Gelatt, C.D. \& Vecci, P.M. (1983). Optimization by simulated annealing. Science, 220, 671-680.

[41] Kennedy, J. \& Eberhart, R. (1997). Particle swarm optimization. IEEE International Conference on Neural Networks, 1, 19421948.

Nurullah Yilmaz is worked as Dr. Research Assistant at Suleyman Demirel University, Isparta. His research interests are functional analysis and non-linear optimization.

Ahmet Sahiner is presently employed as Full Professor at Suleyman Demirel University, Isparta. His research interests are functional analysis, global optimization and approximation theory.

An International Journal of Optimization and Control: Theories \& Applications (http://ijocta.balikesir.edu.tr)

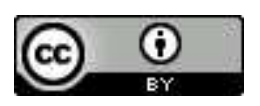

This work is licensed under a Creative Commons Attribution 4.0 International License. The authors retain ownership of the copyright for their article, but they allow anyone to download, reuse, reprint, modify, distribute, and/or copy articles in IJOCTA, so long as the original authors and source are credited. To see the complete license contents, please visit http://creativecommons.org/licenses/by/4.0/. 